\title{
Marginalized Maximum Likelihood for Parameters Estimation of the Three Parameter Weibull Distribution
}

\author{
Ouindllassida Jean-Etienne Ouédraogo ${ }^{1}$, Edoh Katchekpele ${ }^{2} \&$ Simplice Dossou-Gbété $^{3}$ \\ ${ }^{1}$ Laboratoire de Mathématiques Fondamentales et Appliquées (LAMFA), Unit de Formation et Recherche en Sciences et \\ Technologies, Université Norbert ZONGO, B.P. 376 Koudougou, Burkina Faso \\ 2 Département de mathématiques, Faculté des sciences et Techniques, Université de Kara, Kara, TOGO \\ ${ }^{3}$ Laboratoire de Mathématiques et de leurs Applications de Pau (LMAP), UMR 5142 CNRS-Université de Pau et des \\ Pays de 1Adour, Avenue de luniversité, B.P. 576, 64012 Pau cedex (France) Correspondence: Ouindllassida Jean-Etienne \\ Ouédraogo, aboratoire de Mathématiques Fondamentales et Appliquées (LAMFA), Unité de Formation et Recherche en \\ Sciences et Technologies, Université Norbert ZONGO, B.P. 376 Koudougou, Burkina Faso
}

Received: April 15, 2021 Accepted: May 19, 2021 Online Published: June 13, 2021

doi:10.5539/ijsp.v10n4p62 URL: https://doi.org/10.5539/ijsp.v10n4p62

\begin{abstract}
The aims of this paper is to propose a new approach for fitting a three-parameter weibull distribution to data from an independent and identically distributed scheme of sampling. This approach use a likelihood function based on the $n-1$ largest order statistics. Information loss by dropping the first order statistic is then retrieved via an MM-algorithm which will be used to estimate the model's parameters. To examine the properties of the proposed estimators, the associated bias and mean squared error were calculated through Monte Carlo simulations. Subsequently, the performance of these estimators were compared with those of two concurrent methods.
\end{abstract}

Keywords: estimation, likelihood, MM-algorithm, order statistics, three-parameter Weibull model

\section{Introduction}

The Weibull distribution is a continuous probability distribution orginaly used as a model for material breaking strength. Today, it's commonly used to assess product reliability, analyze life data and model failure times. Two versions of the Weibull probability density function (pdf) are in common use: the two parameter pdf and the three parameter pdf. In this paper, our interest will focus on the latest version. A cumulative distribution function (cdf) is indexed by a vector of three real parameters $\theta=(\lambda, \beta, v)$ and is defined as follows:

$$
F(x, \theta)=1-\exp \left\{-\left(\frac{x-v}{\beta}\right)^{\lambda}\right\}
$$

The corresponding probability density function (pdf) is

$$
f(x \mid \theta)=\frac{\lambda}{\beta}\left(\frac{x-v}{\beta}\right)^{\lambda-1} \exp \left\{-\left(\frac{x-v}{\beta}\right)^{\lambda}\right\}
$$

where $\beta>0, \lambda>0$ and $v<x$ are the scale, shape and threshold parameters respectively.

If the threshold parameter $v$ is to estimate, the three-parameter Weibull distribution is non-regular and several undesirable situations can then arise when the maximum likelihood method is used to fit the model to data. About this, Griffiths (1980) point out that the log-likelihood is unbounded when the shape parameter is smaller than unity and the location one closed to the smallest order statistic. In the recent past some several works including those of Cheng and Iles (1990); Cheng (1987); Smith (1985) \& al.Nagatsuka and Balakrishnan (2012) have developed methods that aim to provide with reliable estimators as those based on complete and censored samples.

In this article, we develop an estimation method using the likelihood based on the $n-1$ largest values of a sample of size $n$ from a three-parameter Weibull distribution. A simular likelihood function has been experiment by Etienne Ouindllassida Jean Ouedraogo and Dossou-Gbete (2017) for fitting gamma distribution to its data. But taking into account the lost information linked to the absence of the first order statistic had not been sufficiently clarified. In this paper a section has 
been reserved for this. We provide an MM algorithm as in Lange and Yang (2000) to determine Maximum Likelihood Estimates (MLEs) of $\theta$. The performance of the methodology developed is assessed by a simulation study.

The rest of the paper is organized as follows. The next section describes the proposed method while the third section deals with the minorizing function obtained via an MM approach. Monte-Carlo simulation results are presented in the fourth section. The last section is devoted to a discussion.

\section{The Method}

\subsection{Rationale for the Methodology}

In information theory it is clear that deleting an observation leads to the loss of the information it holds. However, for the precise case of location-scale models family including the Weibull distribution, several works including those of Hill (1963), Griffiths (1980) and Smith (1994) motivate the suppression of the first order statistic. Indeed in Griffiths (1980) it's argued that when traditional likelihood function used, the maximum likelihood is $+\infty$ and is attained when threshold parameter is sufficiently closed to the first order statistic. To get around this problem, a likelihood function marginalized by the first order statistic will be proposed in this paper.

Once the marginalized likelihood function defined, it will be necessary to find a suitable algorithm for the model parameters estimation. In inference statistical litterature several methods are generally used, including Newton-Raphson algorithm, scorring method and MM-algorithm. Regarding this paper the last one has been retained and this choice of the MM-algorithm is strategic. Indeed, in addition to being an efficient tool for estimating model parameters, this algorith$\mathrm{m}$ allows taking into account of information lost when the first order statistic omited in the initialization of the method discussed here. In our concern, we focus on Minorization-Maximization version of the MM approach to computing the argument value where an objective function reaches a maximum, since our goal is to maximize an objective function. This procedure maximize an surrogate function instead of the objective function at each iteration of the algorithm. The first step of a MM-algorithm consists looking for the surrogate function noted Q. This function is also called a minorization function. One of the challenges is to be able to build an easy to manage $Q$ minorization function. To do this, we generally use mathematical inequalities such as the arithmetic or geometric mean inequality, Cauchy-Schwarz inequality, Jensen inequality, minimization via the support hyperplane, etc. It is of course in this stage we will judiciously take into account the information due to the smallest order statistic. For detailled description, reader may refer to the works of Lange(Lange and Yang (2000); Hunter and Lange (2004); Lange (2013)) or Vaida (2005) for convergence proofs.

In the sequel, accronym MosLE method (for "Marginalized order statistics based Likelihood Estimation") will be used to denote the proposed method.

\subsection{Mains Results: An Marginalized Likelihood Function for Three-Parameter Weibull Distribution}

Theorem 2.1. Let $\left(x_{i}\right)_{i=1: n}$ be a sample of size $n$ derived from a three-parameter Weibull distribution and $\theta=(\lambda, \beta, v)$ the unknown parameters vector. Consider $\left(x_{i: n}\right)_{i=1: n}$ is the sequence of the non-decreasing sorted values. An marginalized log-likelihood function based on the $n-1$ largest order statistics is given as follows:

$$
\begin{aligned}
l\left(\theta \mid x_{2: n}, \cdots, x_{n: n}\right) & =\ln (n !)+(n-1) \ln \lambda-(n-1) \lambda \ln \beta-\sum_{r=2}^{n} \ln \left(x_{r: n}-v\right)+\lambda \sum_{r=2}^{n} \ln \left(x_{r: n}-v\right) \\
& -\sum_{r=2}^{n}\left(\frac{x_{r: n}-v}{\beta}\right)^{\lambda}-\left(\frac{x_{2: n}-v}{\beta}\right)^{\lambda}+\ln \left(\exp \left\{\left(\frac{x_{2: n}-v}{\beta}\right)^{\lambda}\right\}-1\right)
\end{aligned}
$$

Proof. Let $\left(X_{i}\right)_{i=1: n}$ be an independent and identically distributed (i.i.d) sequence. Consider $f(x \mid \theta)$ is the marginal density function of Weibull distribution and denote $\left(X_{i: n}\right)_{i=1: n}$ the associated sequence of order statistics. It is well known that the joint density of $\left(X_{i: n}\right)_{i=1: n}$ is

$$
f_{\left(X_{i n}\right)_{i=1: n}}(x \mid \theta)=n ! \prod_{i=1}^{n} f\left(x_{i} \mid \theta\right) 1_{\left\{v \leq x_{1}<x_{2}<\ldots<x_{n}\right\}}(x),
$$

where $x=\left(x_{1}, x_{2}, \cdots, x_{n}\right)$. The joint density of the sequence $\left(X_{i: n}\right)_{i=2: n}$ is therefore defined by

$$
f_{\left(X_{i n}\right)_{i=2: n}}(x \mid \theta)=n ! F\left(x_{2} \mid \theta\right) \prod_{i=2}^{n} f\left(x_{i} \mid \theta\right) 1_{\left\{v \leq x_{2}<x_{3}<\ldots<x_{n}\right\}}(x)
$$

where $F(x \mid \theta)$ is the cumulative distribution function (cdf) of weibull distribution. For detailled exposition of order statistics properties reader may consult the work of David (2003). Viewing the joint density (3) as the likelihood function, the 
Marginalized order statistics based Likelihood function is obtained as follows:

$$
L(x, \theta)=n ! F\left(x_{2: n} \mid \theta\right) \prod_{r=2}^{n} f\left(x_{r: n} \mid \theta\right)
$$

Appealing (1)-(2) and taking logarithms of each side in (4), we complete the proof.

\section{Derivation of a Minorizing Function}

The design of an MM algorithm requires a judicious choice of a minimizing function that is easier to maximize. This minorizing function must be constructed in accordance with the following definition proposed by Lange and Yang (2000).

Definition 3.1. A function $Q\left(\theta \mid \theta^{\prime}\right)$ is said to minorize the function $f(\theta)$ at $\theta^{\prime}$ provided if

$$
\begin{gathered}
f\left(\theta^{\prime}\right)=Q\left(\theta^{\prime} \mid \theta^{\prime}\right) \\
f(\theta) \geq Q\left(\theta \mid \theta^{\prime}\right)
\end{gathered}
$$

for all $\theta$. In Lange and Yang (2000); Hunter and Lange (2004); Lange (2013), it proved that the MM principle has huge advantages as parameters separation, linearization of an optimization problem.

The subsection below is devoted to derive an minorizing function for our log-likelihood function.

\subsection{Design of a Minorizing Function for the Marginalized Log-likelihood}

Let's denote

$$
\begin{gathered}
l_{1}\left(\theta \mid x_{2: n}, \cdots, x_{n: n}\right)=(n-1) \ln \lambda-(n-1) \lambda \ln \beta-\sum_{r=2}^{n} \ln \left(x_{r: n}-v\right)+\lambda \sum_{r=2}^{n} \ln \left(x_{r: n}-v\right) \\
l_{2}\left(\theta \mid x_{2: n}, \cdots, x_{n: n}\right)=-\sum_{r=2}^{n}\left(\frac{x_{r: n}-v}{\beta}\right)^{\lambda}-\left(\frac{x_{2: n}-v}{\beta}\right)^{\lambda} \\
l_{3}\left(\theta \mid x_{2: n}\right)=\ln \left(\exp \left\{\left(\frac{x_{2: n}-v}{\beta}\right)^{\lambda}\right\}-1\right)
\end{gathered}
$$

One has $l\left(\theta \mid x_{2: n}, \cdots, x_{n: n}\right)=\ln (n !)+l_{1}\left(\theta \mid x_{2: n}, \cdots, x_{n: n}\right)+l_{2}\left(\theta \mid x_{2: n}, \cdots, x_{n: n}\right)+l_{3}\left(\theta \mid x_{2: n}\right)$

In the sequel $\theta^{\prime}=\left(\lambda^{\prime}, \beta^{\prime}, v^{\prime}\right)$ will denote a parameters' vector provided and $Q\left(\theta \mid \theta^{\prime}, \ldots\right)$ an minorizing function at $\theta^{\prime}$.

Proposition 3.1. $l_{1}\left(\theta \mid x_{2: n}, \cdots, x_{n: n}\right) \geq Q_{1}\left(\theta \mid \theta^{\prime},\left(x_{i: n}\right)_{i=1: n}\right)$ where

$$
\begin{gathered}
Q_{1}\left(\theta \mid \theta^{\prime},\left(x_{i: n}\right)_{i=1: n}\right)=(n-1) \ln \lambda+\lambda(n-1)\left(1-\log \left(\beta^{\prime}\right)\right) \\
+\lambda\left\{\sum_{r=2}^{n} \ln \left(x_{r: n}-v^{\prime}\right)+\sum_{r=2}^{n}\left[\frac{x_{1: n}-v^{\prime}}{x_{r: n}-v^{\prime}}\right]\right\}-\frac{\lambda^{2}}{2 \lambda^{\prime}}\left\{(n-1)+\sum_{r=2}^{n}\left[\frac{x_{1: n}-v^{\prime}}{x_{r: n}-v^{\prime}}\right]\right\} \\
-\frac{\left(x_{1: n}-v^{\prime}\right)^{3}}{\left(x_{1: n}-v\right)^{2}} \frac{\lambda^{\prime}}{2} \sum_{r=2}^{n} \frac{1}{x_{r: n}-v^{\prime}}+\sum_{r=2}^{n} \frac{v-v^{\prime}}{x_{r: n}-v^{\prime}}-(n-1) \lambda^{\prime} \frac{\beta^{2}}{2 \beta^{\prime 2}}-\sum_{r=2}^{n} \ln \left(x_{r: n}-v^{\prime}\right)
\end{gathered}
$$

Proof. See AppendixA.2

Proposition 3.2. Let $\left(x_{i: n}\right)_{i=1: n}$ be a sequence of the non-decreasing sorted values derived from the three-parameter Weibull distribution with the parameters vectors $\theta=(\lambda, \beta, v)$. The following statement hold: $l_{2}\left(\theta \mid x_{2: n}, \cdots, x_{n: n}\right) \geq$ $Q_{2}\left(\theta \mid \theta^{\prime},\left(x_{i: n}\right)_{i=1: n}\right)$ where 


$$
\begin{gathered}
Q_{2}\left(\theta \mid \theta^{\prime},\left(x_{i: n}\right)_{i=1: n}\right)=-\frac{1}{3} \sum_{r=2}^{n} \exp \left\{3 \lambda\left[\ln \left(x_{r: n}-v^{\prime}\right)-2-\ln \beta^{\prime}\right]+\frac{3 \lambda^{2}}{\lambda^{\prime}}\right\} \\
-\frac{1}{3} \exp \left\{3 \lambda\left[\ln \left(x_{2: n}-v^{\prime}\right)-2-\ln \beta^{\prime}\right]+\frac{3 \lambda^{2}}{\lambda^{\prime}}\right\}-\frac{n}{3} \exp \left\{\frac{3}{\beta^{2}} \frac{\beta^{\prime 2} \lambda^{\prime}}{2}\right\} \\
-\frac{1}{3} \sum_{r=2}^{n} \exp \left\{\frac{3 \lambda^{\prime}\left(x_{r: n}-v\right)^{2}}{2\left(x_{r: n}-v^{\prime}\right)^{2}}\right\}-\frac{1}{3} \exp \left\{\frac{3 \lambda^{\prime}\left(x_{2: n}-v\right)^{2}}{2\left(x_{2: n}-v^{\prime}\right)^{2}}\right\} \\
-\frac{1}{3} \sum_{r=2}^{n} \exp \left\{-2 \lambda^{\prime} \ln \left(x_{r: n}-v^{\prime}\right)+3 \lambda^{\prime}+2 \lambda^{\prime} \ln \beta^{\prime}\right\}-\frac{2}{3} \sum_{r=2}^{n} \exp \left\{\lambda^{\prime} \ln \left(x_{r: n}-v^{\prime}\right)-\frac{3}{2} \lambda^{\prime}-\lambda^{\prime} \ln \beta^{\prime}\right\} \\
-\frac{1}{3} \exp \left\{-2 \lambda^{\prime} \ln \left(x_{2: n}-v^{\prime}\right)+3 \lambda^{\prime}+2 \lambda^{\prime} \ln \beta^{\prime}\right\}-\frac{2}{3} \exp \left\{\lambda^{\prime} \ln \left(x_{2: n}-v^{\prime}\right)-\frac{3}{2} \lambda^{\prime}-\lambda^{\prime} \ln \beta^{\prime}\right\}
\end{gathered}
$$

Proof. See AppendixA.3

Proposition 3.3. $l_{3}\left(\theta \mid x_{2: n}\right) \geq Q_{3}\left(\theta \mid \theta^{\prime}, x_{1: n}, x_{2: n}\right)$ where

$$
\begin{gathered}
Q_{3}\left(\theta \mid \theta^{\prime}, x_{1: n}, x_{2: n}\right)=a \lambda\left\{\ln \left(x_{2: n}-v^{\prime}\right)-\ln \left(\beta^{\prime}\right)+1+\left[\frac{x_{1: n}-v^{\prime}}{x_{2: n}-v^{\prime}}\right]\right\}-a\left[1+\frac{x_{1: n}-v^{\prime}}{x_{2: n}-v^{\prime}}\right] \frac{\lambda^{2}}{2 \lambda^{\prime}} \\
+\frac{b \exp \left[3 \lambda \ln \left(x_{2: n}-v^{\prime}\right)-6 \lambda+\frac{3 \lambda^{2}}{\lambda^{\prime}}-3 \lambda \ln \beta^{\prime}\right]}{3 \exp \left(2 \lambda^{\prime} \ln \left(x_{2: n}-v^{\prime}\right)-3 \lambda^{\prime}-2 \lambda^{\prime} \ln \beta^{\prime}\right)}-a\left[\frac{x_{1: n}-v^{\prime}}{x_{2: n}-v^{\prime}}\right] \frac{\lambda^{\prime}\left(x_{1: n}-v^{\prime}\right)^{2}}{2\left(x_{1: n}-v\right)^{2}} \\
+\frac{b \exp \left(\left(x_{2: n}-v\right)^{2} \frac{3 \lambda^{\prime}}{2\left(x_{2: n}-v^{\prime}\right)^{2}}\right)}{3} \exp \left(\lambda^{\prime} \ln \left(x_{2: n}-v^{\prime}\right)-\frac{3}{2} \lambda^{\prime}-\lambda^{\prime} \log \beta^{\prime}\right) \\
+\frac{b \exp \left(\frac{3}{\beta^{2}} \frac{\beta^{\prime 2} \lambda^{\prime}}{2}\right)}{3} \exp \left(\lambda^{\prime} \ln \left(x_{2: n}-v^{\prime}\right)-\frac{3}{2} \lambda^{\prime}-\lambda^{\prime} \ln \beta^{\prime}\right)-a \lambda^{\prime} \frac{\beta^{2}}{2 \beta^{\prime 2}}
\end{gathered}
$$

and the constant terms $a, b$ and $c$ are given by

$$
\begin{gathered}
a=\frac{\Pi^{2}\left(\theta^{\prime} \mid x_{2: n}\right) \exp \left(\Pi\left(\theta^{\prime} \mid x_{2: n}\right)\right)}{\left[\exp \left(\Pi\left(\theta^{\prime} \mid x_{2: n}\right)\right)-1\right]^{2}}+\frac{\Pi\left(\theta^{\prime} \mid x_{2: n}\right) \exp \left(\Pi\left(\theta^{\prime} \mid x_{2: n}\right)\right)}{\exp \left(\Pi\left(\theta^{\prime} \mid x_{2: n}\right)\right)-1} \\
b=-\frac{\Pi\left(\theta^{\prime} \mid x_{2: n}\right) \exp \left(\Pi\left(\theta^{\prime} \mid x_{2: n}\right)\right)}{\exp \left(\Pi\left(\theta^{\prime} \mid x_{2: n}\right)\right)-1}
\end{gathered}
$$

and

$$
c=\log \left(\exp \left(\Pi\left(\theta^{\prime} \mid x_{2: n}\right)\right)-1\right)-a \log \left(\Pi\left(\theta^{\prime} \mid x_{2: n}\right)\right)-b \Pi\left(\theta^{\prime} \mid x_{2: n}\right)
$$

with $\Pi\left(\theta \mid x_{2: n}\right)=\left(\frac{x_{2: n}-v}{\beta}\right)^{\lambda}$

Proof. See AppendixA.4

Since

$$
l\left(\theta \mid x_{2: n}, \cdots, x_{n: n}\right)=\ln (n !)+l_{1}\left(\theta \mid x_{2: n}, \cdots, x_{n: n}\right)+l_{2}\left(\theta \mid x_{2: n}, \cdots, x_{n: n}\right)+l_{3}\left(\theta \mid x_{2: n}\right)
$$

one has:

$l\left(\theta \mid x_{2: n}, \cdots, x_{n: n}\right) \geq \ln (n !)+Q\left(\theta \mid \theta^{\prime},\left(x_{i: n}\right)_{i=1: n}\right)$, where $Q\left(\theta \mid \theta^{\prime},\left(x_{i: n}\right)_{i=1: n}\right)=Q_{1}\left(\theta \mid \theta^{\prime},\left(x_{i: n}\right)_{i=1: n}\right)+Q_{2}\left(\theta \mid \theta^{\prime},\left(x_{i: n}\right)_{i=1: n}\right)+$ $Q_{3}\left(\theta \mid \theta^{\prime}, x_{1: n}, x_{2: n}\right)$

with $Q_{1}\left(\theta \mid \theta^{\prime},\left(x_{i: n}\right)_{i=1: n}\right), Q_{2}\left(\theta \mid \theta^{\prime},\left(x_{i: n}\right)_{i=1: n}\right)$ and $Q_{3}\left(\theta \mid \theta^{\prime}, x_{1: n}, x_{2: n}\right)$ given precedently. 


\subsection{Some Properties of the Obtained Minorizing Function}

After rearangements, first derivatives of the $Q$-function are obtained as follow:

$$
\begin{aligned}
& \frac{\partial Q\left(\theta \mid \theta^{\prime},\left(x_{i: n}\right)_{i=1: n}\right)}{\partial v}=-\lambda^{\prime}\left(\frac{x_{1: n}-v^{\prime}}{x_{1: n}-v}\right)^{3}\left\{\sum_{r=2}^{n} \frac{1}{x_{r: n}-v^{\prime}}+\frac{a}{x_{2: n}-v^{\prime}}\right\} \\
& +\sum_{r=2}^{n} \frac{\lambda^{\prime}\left(x_{r: n}-v\right)}{\left(x_{r: n}-v^{\prime}\right)^{2}} \exp \left\{\frac{3 \lambda^{\prime}\left(x_{r: n}-v\right)^{2}}{2\left(x_{r: n}-v^{\prime}\right)^{2}}\right\} \\
& \frac{\lambda^{\prime}\left(x_{2: n}-v\right)}{\left(x_{2: n}-v^{\prime}\right)^{2}} \exp \left(\frac{3 \lambda^{\prime}\left(x_{2: n}-v\right)^{2}}{2\left(x_{2: n}-v^{\prime}\right)^{2}}\right)\left\{1-b \exp \left(\lambda^{\prime} \ln \left(x_{2: n}-v^{\prime}\right)-\frac{3}{2} \lambda^{\prime}-\lambda^{\prime} \ln \beta^{\prime}\right)\right\} \\
& +\sum_{r=2}^{n} \frac{1}{x_{r: n}-v^{\prime}} \\
& \frac{\partial Q\left(\theta \mid \theta^{\prime},\left(x_{i: n}\right)_{i=1: n}\right)}{\partial \lambda}=\frac{(n-1)}{\lambda}-\frac{\lambda}{\lambda^{\prime}}\left\{(n-1)+\sum_{r=2}^{n}\left[\frac{x_{1: n}-v^{\prime}}{x_{r: n}-v^{\prime}}\right]+a\left[1+\frac{x_{1: n}-v^{\prime}}{x_{2: n}-v^{\prime}}\right]\right\} \\
& -\sum_{r=2}^{n}\left\{\ln \left(x_{r: n}-v^{\prime}\right)-2-\ln \beta^{\prime}+\frac{2 \lambda}{\lambda^{\prime}}\right\} \exp \left\{3 \lambda\left[\ln \left(x_{r: n}-v^{\prime}\right)-2-\ln \beta^{\prime}\right]+\frac{3 \lambda^{2}}{\lambda^{\prime}}\right\} \\
& -\left\{\ln \left(x_{2: n}-v^{\prime}\right)-2-\log \beta^{\prime}+\frac{2 \lambda}{\lambda^{\prime}}\right\} \exp \left\{3 \lambda\left[\ln \left(x_{2: n}-v^{\prime}\right)-2-\ln \beta^{\prime}\right]+\frac{3 \lambda^{2}}{\lambda^{\prime}}\right\} \\
& +\frac{b\left\{\ln \left(x_{2: n}-v^{\prime}\right)-2+\frac{2 \lambda}{\lambda^{\prime}}-\ln \beta^{\prime}\right\} \exp \left[3 \lambda \ln \left(x_{2: n}-v^{\prime}\right)-6 \lambda+\frac{3 \lambda^{2}}{\lambda^{\prime}}-3 \lambda \ln \beta^{\prime}\right]}{\exp \left(2 \lambda^{\prime} \ln \left(x_{2: n}-\nu^{\prime}\right)-3 \lambda^{\prime}-2 \lambda^{\prime} \ln \beta^{\prime}\right)} \\
& +a\left\{\ln \left(x_{2: n}-v^{\prime}\right)-\ln \left(\beta^{\prime}\right)+1+\frac{x_{1: n}-v^{\prime}}{x_{2: n}-v^{\prime}}\right\}+(n-1)\left(1-\ln \left(\beta^{\prime}\right)\right) \\
& +\sum_{r=2}^{n} \ln \left(x_{r: n}-v^{\prime}\right)+\sum_{r=2}^{n}\left[\frac{x_{1: n}-v^{\prime}}{x_{r: n}-v^{\prime}}\right] \\
& \frac{\partial Q\left(\theta \mid \theta^{\prime},\left(x_{i: n}\right)_{i=1: n}\right)}{\partial \beta}=-\lambda^{\prime} \beta\left[\frac{n-1+a}{\beta^{\prime 2}}\right] \\
& +\frac{\beta^{2} \lambda^{\prime}}{\beta^{3}} \exp \left(\frac{3}{\beta^{2}} \frac{\beta^{2} \lambda^{\prime}}{2}\right)\left\{n-b \exp \left(\lambda^{\prime} \ln \left(x_{2: n}-v^{\prime}\right)-\frac{3}{2} \lambda^{\prime}-\lambda^{\prime} \ln \beta^{\prime}\right)\right\}
\end{aligned}
$$

Proposition 3.4. The function $\theta \rightarrow Q\left(\theta \mid \theta^{\prime},\left(x_{i: n}\right)_{i=1: n}\right)$ admits a unique global maximum for any fixed $\theta^{\prime}$.

Proof. Hessian matrix of the function $\theta \rightarrow Q\left(\theta \mid \theta^{\prime},\left(x_{i: n}\right)_{i=1: n}\right)$ is diagonal with nonzero components as follows:

$$
\begin{gathered}
\quad \frac{\partial^{2} Q\left(\theta \mid \theta^{\prime},\left(x_{i: n}\right)_{i=1: n}\right)}{\partial v^{2}}=-\frac{3}{\left(x_{1: n}-v\right)^{4}} \lambda^{\prime} \sum_{r=2}^{n} \frac{x_{1: n}-v^{\prime}}{x_{r: n}-v^{\prime}}-\frac{3 a}{\left(x_{1: n}-v\right)^{4}} \frac{\lambda^{\prime}\left(x_{1: n}-v^{\prime}\right)^{3}}{x_{2: n}-v^{\prime}} \\
-\sum_{r=2}^{n} \frac{\lambda^{\prime}}{\left(x_{r: n}-v^{\prime}\right)^{2}} \exp \left\{\frac{3 \lambda^{\prime}\left(x_{r: n}-v\right)^{2}}{2\left(x_{r: n}-v^{\prime}\right)^{2}}\right\}-3 \sum_{r=2}^{n}\left[\frac{\lambda^{\prime}\left(x_{r: n}-v\right)}{\left(x_{r: n}-v^{\prime}\right)^{2}}\right]^{2} \exp \left\{\frac{3 \lambda^{\prime}\left(x_{r: n}-v\right)^{2}}{2\left(x_{r: n}-v^{\prime}\right)^{2}}\right\} \\
-\frac{\lambda^{\prime}}{\left(x_{2: n}-v^{\prime}\right)^{2}} \exp \left\{\frac{3 \lambda^{\prime}\left(x_{2: n}-v\right)^{2}}{2\left(x_{2: n}-v^{\prime}\right)^{2}}\right\}-3\left[\frac{\lambda^{\prime}\left(x_{2: n}-v\right)}{\left(x_{2: n}-v^{\prime}\right)^{2}}\right]^{2} \exp \left\{\frac{3 \lambda^{\prime}\left(x_{2: n}-v\right)^{2}}{2\left(x_{2: n}-v^{\prime}\right)^{2}}\right\} \\
+\frac{b \lambda^{\prime}}{\left(x_{2: n}-v^{\prime}\right)^{2}} \exp \left(\frac{3 \lambda^{\prime}\left(x_{2: n}-v\right)^{2}}{2\left(x_{2: n}-v^{\prime}\right)^{2}}\right) \exp \left(\lambda^{\prime} \ln \left(x_{2: n}-v^{\prime}\right)-\frac{3}{2} \lambda^{\prime}-\lambda^{\prime} \ln \beta^{\prime}\right) \\
+3 b\left\{\frac{\lambda^{\prime}\left(x_{2: n}-v\right)}{\left(x_{2: n}-v^{\prime}\right)^{2}}\right\}^{2} \exp \left(\frac{3 \lambda^{\prime}\left(x_{2: n}-v\right)^{2}}{2\left(x_{2: n}-v^{\prime}\right)^{2}}\right) \exp \left(\lambda^{\prime} \ln \left(x_{2: n}-v^{\prime}\right)-\frac{3}{2} \lambda^{\prime}-\lambda^{\prime} \ln \beta^{\prime}\right)
\end{gathered}
$$




$$
\begin{aligned}
& \frac{\partial^{2} Q\left(\theta \mid \theta^{\prime},\left(x_{i: n}\right)_{i=1: n}\right)}{\partial \beta^{2}}=-\frac{(n-1) \lambda^{\prime}}{\beta^{\prime 2}}-a \lambda^{\prime} \frac{\beta}{\beta^{\prime 2}} \\
& -\frac{3 n \beta^{2} \lambda^{\prime}}{\beta^{4}} \exp \left\{\frac{3}{\beta^{2}} \frac{\beta^{2} \lambda^{\prime}}{2}\right\}-3 n\left(\frac{\beta^{2} \lambda^{\prime}}{\beta^{3}}\right)^{2} \exp \left\{\frac{3}{\beta^{2}} \frac{\beta^{2} \lambda^{\prime}}{2}\right\} \\
& +3 b\left\{\frac{\beta^{2} \lambda^{\prime}}{\beta^{4}}+\left(\frac{\beta^{\prime 2} \lambda^{\prime}}{\beta^{3}}\right)^{2}\right\} \exp \left(\frac{3}{\beta^{2}} \frac{\beta^{\prime 2} \lambda^{\prime}}{2}\right) \exp \left(\lambda^{\prime} \ln \left(x_{2: n}-v^{\prime}\right)-\frac{3}{2} \lambda^{\prime}-\lambda^{\prime} \ln \beta^{\prime}\right) \\
& \frac{\partial^{2} Q\left(\theta \mid \theta^{\prime},\left(x_{i: n}\right)_{i=1: n}\right)}{\partial \lambda^{2}}=-\frac{(n-1)}{\lambda^{2}}-\frac{1}{\lambda^{\prime}}\left\{(n-1)+\sum_{r=2}^{n}\left[\frac{x_{1: n}-v^{\prime}}{x_{r: n}-v^{\prime}}\right]\right\} \\
& -\sum_{r=2}^{n} \frac{2}{\lambda^{\prime}} \exp \left\{3 \lambda\left[\ln \left(x_{r: n}-v^{\prime}\right)-2-\ln \beta^{\prime}\right]+\frac{3 \lambda^{2}}{\lambda^{\prime}}\right\} \\
& -3 \sum_{r=2}^{n}\left\{\ln \left(x_{r: n}-v^{\prime}\right)-2-\ln \beta^{\prime}+\frac{2 \lambda}{\lambda^{\prime}}\right\}^{2} \exp \left\{3 \lambda\left[\ln \left(x_{r: n}-v^{\prime}\right)-2-\ln \beta^{\prime}\right]+\frac{3 \lambda^{2}}{\lambda^{\prime}}\right\} \\
& -\frac{2}{\lambda^{\prime}} \exp \left\{3 \lambda\left[\ln \left(x_{2: n}-v^{\prime}\right)-2-\ln \beta^{\prime}\right]+\frac{3 \lambda^{2}}{\lambda^{\prime}}\right\} \\
& -3\left\{\ln \left(x_{2: n}-v^{\prime}\right)-2-\ln \beta^{\prime}+\frac{2 \lambda}{\lambda^{\prime}}\right\}^{2} \exp \left\{3 \lambda\left[\ln \left(x_{2: n}-v^{\prime}\right)-2-\ln \beta^{\prime}\right]+\frac{3 \lambda^{2}}{\lambda^{\prime}}\right\} \\
& -a\left[1+\frac{x_{1: n}-v^{\prime}}{x_{2: n}-v^{\prime}}\right] \frac{1}{\lambda^{\prime}}+\frac{\frac{2 b}{\lambda^{\prime}} \exp \left[3 \lambda \ln \left(x_{2: n}-\nu^{\prime}\right)-6 \lambda+\frac{3 \lambda^{2}}{\lambda^{\prime}}-3 \lambda \ln \beta^{\prime}\right]}{\exp \left(2 \lambda^{\prime} \ln \left(x_{2: n}-v^{\prime}\right)-3 \lambda^{\prime}-2 \lambda^{\prime} \ln \beta^{\prime}\right)} \\
& +\frac{3 b\left\{\ln \left(x_{2: n}-v^{\prime}\right)-2+\frac{2 \lambda}{\lambda^{\prime}}-\ln \beta^{\prime}\right\}^{2} \exp \left[3 \lambda \ln \left(x_{2: n}-v^{\prime}\right)-6 \lambda+\frac{3 \lambda^{2}}{\lambda^{\prime}}-3 \lambda \ln \beta^{\prime}\right]}{\exp \left(2 \lambda^{\prime} \ln \left(x_{2: n}-\nu^{\prime}\right)-3 \lambda^{\prime}-2 \lambda^{\prime} \ln \beta^{\prime}\right)}
\end{aligned}
$$

It's clear that all components are negatives since $a$ and $b$ are positive and negative respectively. Thus, the Hessian matrix is negative-semidefinite. This complete the proof.

Require: $\quad k, \theta_{k}={ }^{t}\left(\lambda_{k}, \beta_{k}, v_{k}\right)$

Output: $\quad k+1, \theta_{k+1}={ }^{t}\left(\lambda_{k+1}, \beta_{k+1}, v_{k+1}\right)$

Do: until a stopping criteria is fulfilled:

- $\lambda\left(\theta_{k+1}\right)={ }^{t}\left(\lambda, \beta_{k}, v_{k}\right)$

$$
\lambda_{k+1}=\arg \max _{\lambda>0}\left\{Q\left(\lambda\left(\theta_{k+1}\right) \mid \theta_{k}\right)\right\}
$$

$\theta_{k}^{\prime}={ }^{t}\left(\lambda_{k+1}, \beta_{k}, v_{k}\right)$,

- $\beta\left(\theta_{k+1}\right)={ }^{t}\left(\lambda_{k+1}, \beta, v_{k}\right)$

$\beta_{k+1}=\arg \max _{\beta>0}\left\{Q\left(\beta\left(\theta_{k+1}\right) \mid \theta_{k}^{\prime}\right)\right\}$

$\theta_{k}^{\prime \prime}={ }^{t}\left(\lambda_{k+1}, \beta_{k+1}, v_{k}\right)$,

- $v\left(\theta_{k+1}\right)={ }^{t}\left(\lambda_{k+1}, \beta_{k+1}, v\right)$

$v_{k+1}=\arg \max _{v<x_{1 \cdot n}}\left\{Q\left(v\left(\theta_{k+1}\right) \mid \theta_{k}^{\prime \prime}\right)\right\}$

$k=k+1$

End:

Algorithm 1: Parameters' updating scheme 


\subsection{Updating Scheme}

\subsubsection{Updating Procedure for the Parameter $v$}

For ease of notations, one can write $\frac{\partial Q\left(\theta \mid \theta^{\prime},\left(x_{i n}\right)_{i=1: n}\right)}{\partial v}=k\left(v \mid \theta^{\prime}\right)$ where $\frac{\partial Q\left(\theta \mid \theta^{\prime},\left(x_{i n}\right)_{i=1: n}\right)}{\partial v}$ is given in subsection 3.2 .

To solve the equation $\frac{\partial Q\left(\theta \mid \theta^{\prime},\left(x_{i n}\right)_{i=1: n}\right)}{\partial v}=0$ is equivalent to $k\left(v \mid \theta^{\prime}\right)=0$. It is clear that $k$ is a monotonically decreasing function on the domain ] $-\infty, x_{1: n}$ [ since $a \geq 0, b \leq 0$ and $\left(x_{r: n}-v^{\prime}\right)>0$ for $r \geq 1$. Moreover $\lim _{v \rightarrow x_{1: n}} k\left(v \mid \theta^{\prime}\right)=-\infty$ and $\lim _{v \rightarrow+\infty} k\left(v \mid \theta^{\prime}\right)=+\infty$. It comes that equation $k\left(v \mid \theta^{\prime}\right)=0$ admits an unique solution in $]-\infty, x_{1: n}[$ as well as the equation $\frac{\partial Q\left(\theta \mid \theta^{\prime},\left(x_{i n}\right)_{i=1: n}\right)}{\partial v}=0$.

\subsubsection{Updating procedure for the parameter $\beta$}

One can notices that

$$
\frac{\partial Q\left(\theta \mid \theta^{\prime},\left(x_{i: n}\right)_{i=1: n}\right)}{\partial \beta}=-\frac{1}{\beta^{3}} g\left(\beta \mid \theta^{\prime}\right)
$$

with

$$
\begin{aligned}
g\left(\beta \mid \theta^{\prime}\right) & =\lambda^{\prime}\left[\frac{n-1+a}{\beta^{2}}\right] \beta^{4} \\
& -\beta^{\prime 2} \lambda^{\prime} \exp \left(\frac{3}{\beta^{2}} \frac{\beta^{2} \lambda^{\prime}}{2}\right)\left\{n-b \exp \left(\lambda^{\prime} \ln \left(x_{2: n}-v^{\prime}\right)-\frac{3}{2} \lambda^{\prime}-\lambda^{\prime} \ln \beta^{\prime}\right)\right\}
\end{aligned}
$$

The assertion $\frac{\partial Q\left(\theta \mid \theta^{\prime},\left(x_{i n}\right)_{i=1: n}\right)}{\partial \beta}=0$ holds if and only if $g\left(\beta \mid \theta^{\prime}\right)=0$, the latter being equivalent to finding root of the function $\beta \rightarrow g\left(\beta \mid \theta^{\prime}\right)$. The derivative $g^{\prime}\left(\beta \mid \theta^{\prime}\right)$ of this function is

$$
\begin{aligned}
g^{\prime}\left(\beta \mid \theta^{\prime}\right) & =4 \lambda^{\prime}\left[\frac{n-1+a}{\beta^{\prime 2}}\right] \beta^{3} \\
+ & \frac{3 \beta^{\prime 4} \lambda^{\prime 2}}{\beta^{3}} \exp \left(\frac{3}{\beta^{2}} \frac{\beta^{2} \lambda^{\prime}}{2}\right)\left\{n-b \exp \left(\lambda^{\prime} \ln \left(x_{2: n}-v^{\prime}\right)-\frac{3}{2} \lambda^{\prime}-\lambda^{\prime} \ln \beta^{\prime}\right)\right\}
\end{aligned}
$$

since $b<0, g^{\prime}\left(\beta \mid \theta^{\prime}\right) \geq 0$ and then the function $\beta \rightarrow g\left(\beta \mid \theta^{\prime}\right)$ is monotonically increasing on the domain $] 0,+\infty[$. Moreover $\lim _{\beta \rightarrow 0} g\left(\beta \mid \theta^{\prime}\right)=-\infty$ and $\lim _{\beta \rightarrow+\infty} g\left(\beta \mid \theta^{\prime}\right)=+\infty$. Thus the equation $g\left(\beta \mid \theta^{\prime}\right)=0$ has an unique solution in ] $0,+\infty\left[\right.$ and the same for the equation $\frac{\partial Q\left(\theta \mid \theta^{\prime},\left(x_{i n}\right)_{i=1: n}\right)}{\partial \beta}=0$.

\subsubsection{Updating Procedure for the Parameter $\lambda$}

For ease of use let us denote $h\left(\lambda \mid \theta^{\prime}\right)=\frac{\partial Q\left(\theta \mid \theta^{\prime},\left(x_{i n}\right)_{i=1: n}\right)}{\partial \lambda}$ given in subsection 3.2. Then the first derivative of the function $\lambda \rightarrow h\left(\lambda \mid \theta^{\prime}\right)$ is $h^{\prime}\left(\lambda \mid \theta^{\prime}\right)=\frac{\partial^{2} Q\left(\theta \mid \theta^{\prime},\left(x_{i n}\right)_{i=1: n}\right)}{\partial \lambda^{2}}$. It is shown in Proposition 3.2 that $h^{\prime}\left(\lambda \mid \theta^{\prime}\right) \leq 0$ for all $\lambda$ in $] 0,+\infty[$. Then, the function $\lambda \rightarrow h\left(\lambda \mid \theta^{\prime}\right)$ is monotonically decreasing on the domain $] 0,+\infty[$. Moreover, without difficulty one must see that $\lim _{\lambda \rightarrow 0} h\left(\lambda \mid \theta^{\prime}\right)=+\infty$ and $\lim _{\lambda \rightarrow+\infty} h\left(\lambda \mid \theta^{\prime}\right)=-\infty$. Thus the equation $h\left(\lambda \mid \theta^{\prime}\right)=0$ has an unique solution in $] 0,+\infty[$.

\section{Simulation Study}

In this sectio, we use Monte Carlo simulations to evaluate the performance of the estimators provided by the MosLE method. We use an sampling design comparable to that of Nagatsuka et al. (2013). As in Nagatsuka et al. (2013), the following values of the shape parameter $\lambda: 0.5,1.0,2.0,3.0$, and 4.0 are selected when the location and the scale parameters are fixed to $v=0$ and $\beta=1$ respectively. The sample size is taken to be 20,50 and 100 . The obtained results are compared to those reported in the paper by Nagatsuka et al. (2013), for Location and Scale Parameters Free Maximum Likelihood method (LSPF-MLE) and the Bayesian Likelihood (BLE) method proposed in Hall and Wang (2005). All computations were carred out with R computing environment (R Core Team, R Core Team (2015)).

\subsection{Graphical Analysis of the Simulation Study Results}

As announced earlier, in this section we compare the results of simulations of the proposed method with those of the LSPF-MLE proposed in Nagatsuka et al. (2013) and BLE in Hall and Wang (2005). The discussion is based on the bias and the root mean squared error estimated(RMSE). 

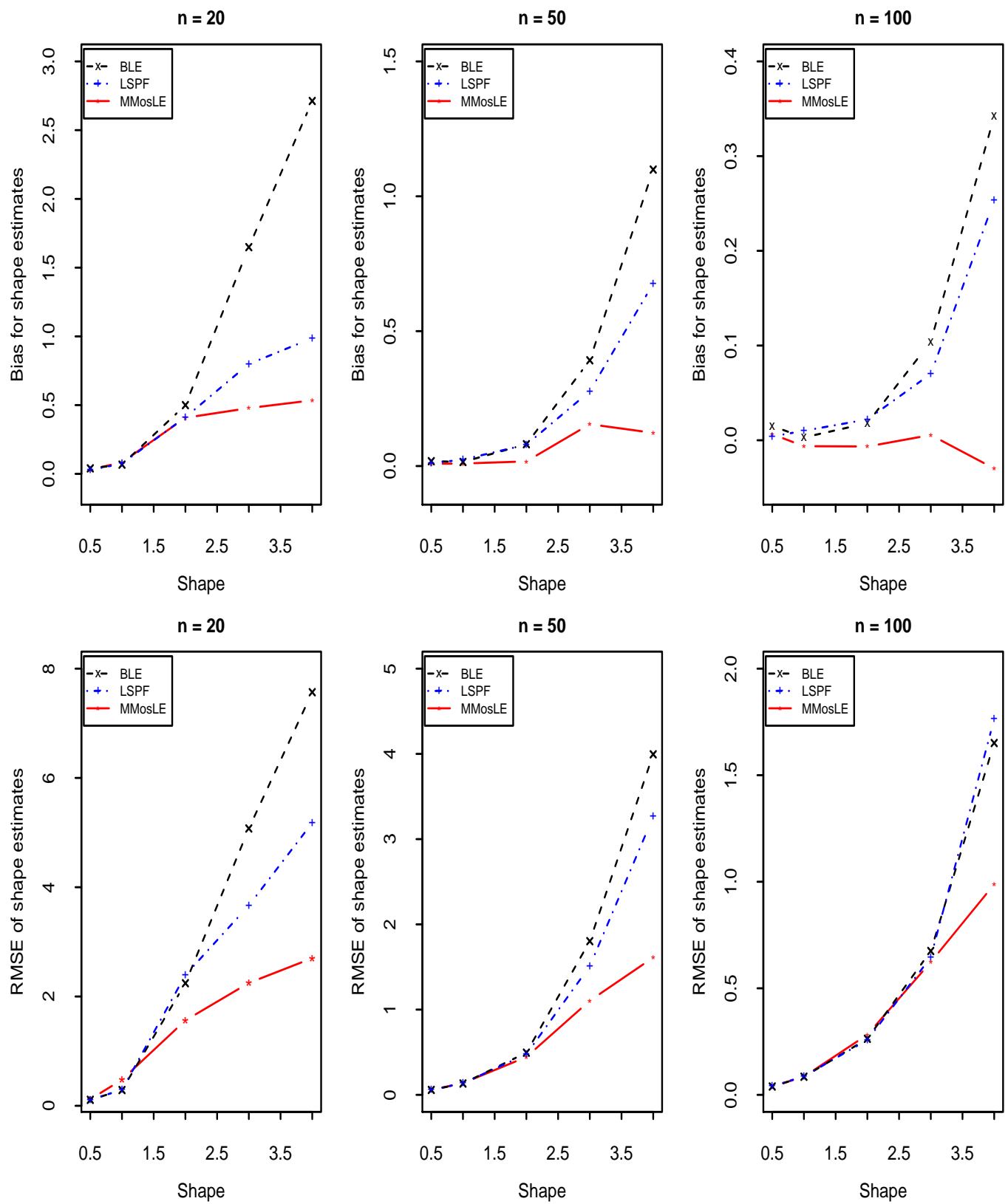

Figure 1. Bias and RMSE for shape parameter estimates based on 1000 simulated samples

The bias of the shape parameter estimate is positive for the three methods in competition and increases with the theoretical value of this parameter. Proof that the three methods overestimate the shape parameter. For small values of the shape parameter $(\lambda \leq 1)$, the magnitude of the bias in the estimate of the shape parameter is similar for all three methods. However, the bias decreases more rapidly in the case of the method proposed here as the sample size increases. Moreover, the analysis of the graph suggests that the estimator proposed by the MosLE method is more stable than those of the other methods within the meaning of the RMSE. 

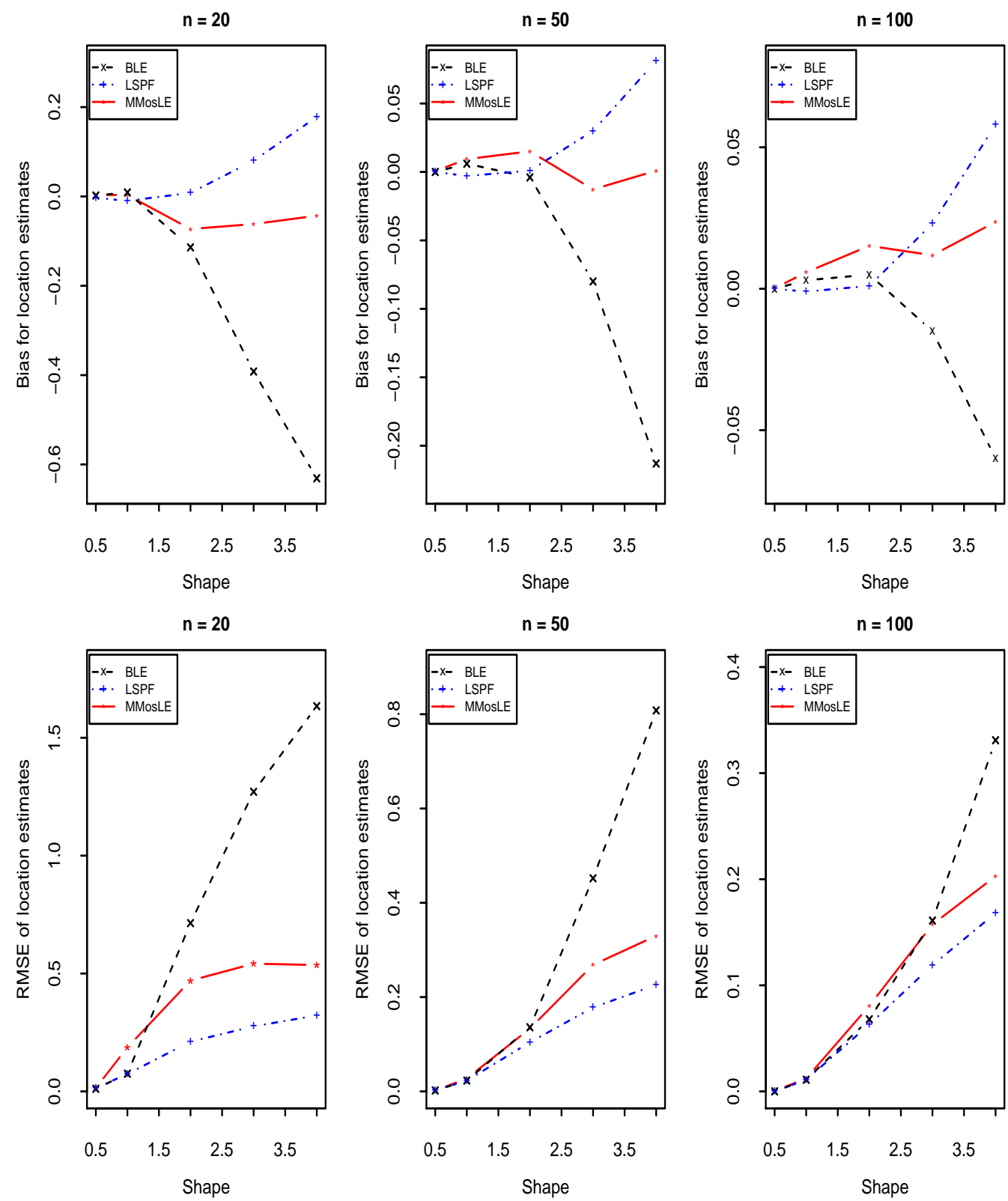

Figure 2. Bias and RMSE for location parameter estimates based on 1000 simulated samples

When sample size is small the proposed method underestimates the location parameter for large value of the shape parameter as the BL method while the LSFP method overestimates the location. For value of the shape parameter larger than 1.5, MosLE estimator provide the smallest bias of the location parameter among the methods in competition. The graphics suggest also the magnitude of the RMSE is comparable between the LSFP method and our method and outperform the BL method. 

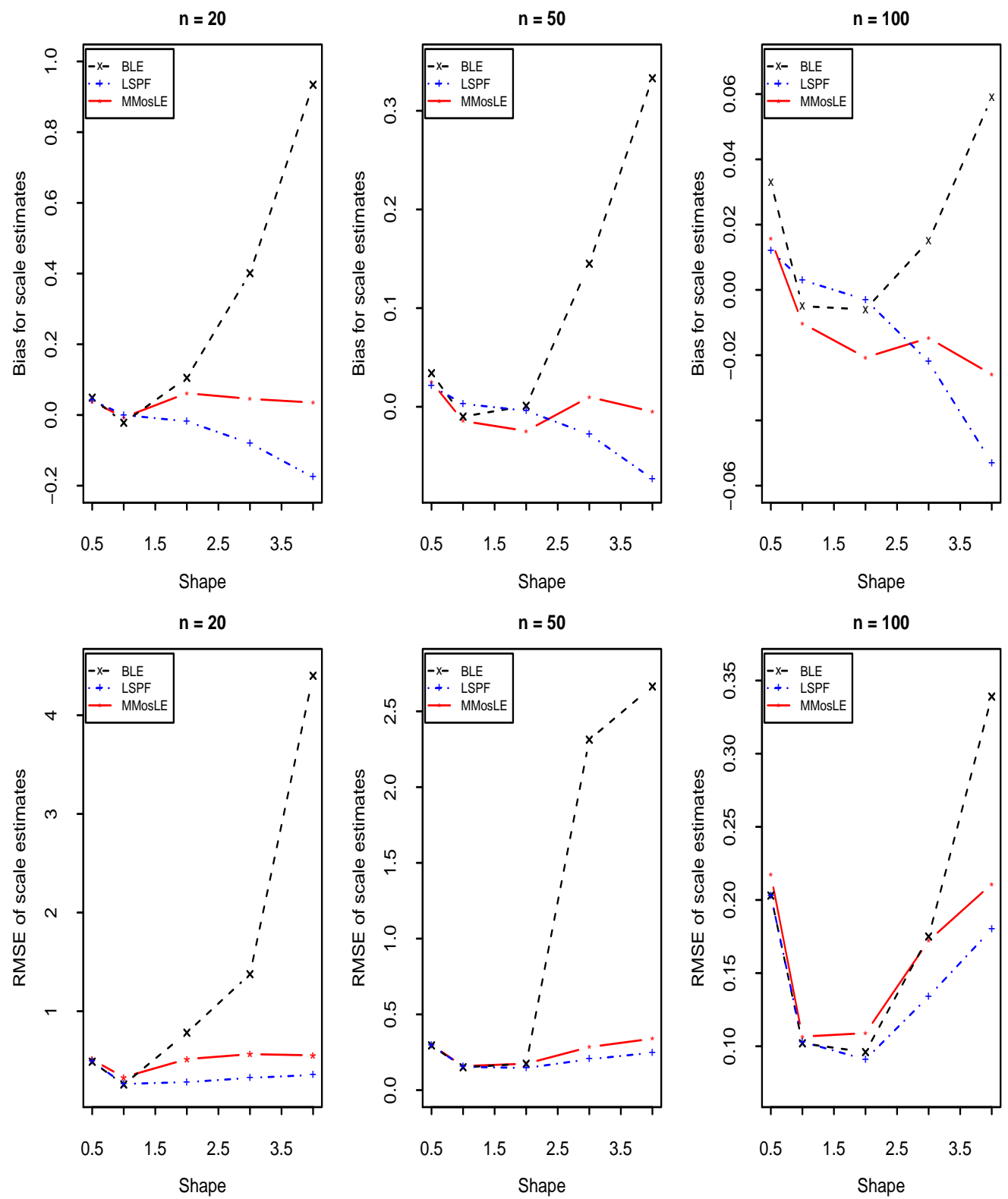

Figure 3. Bias and RMSE for scale parameter estimates based on 1000 simulated samples

The estimator of the scale parameter provided by the MosLE method appears to be more biased than those of the other two methods. However, the magnitude of RMSE is similar for all three methods and increases with the theoretical value of the shape parameter. It can also be noted that the RMSE decreases for the three methods as the sample size increases.

\section{Concluding Remarks}

In this paper we propose an approach based on an marginalised likelihood function to estimate the parameters of the threeparameters Weibull distribution. To maximise the obtained likelihood function, an MM-algorithm is used. Efficiency of the obtained estimator is compared with those of two alternative methods: the location and scale parameters free maximum likelihood estimators (LSPF-MLE) method proposed by Nagatsuka et al. (2013), and the Bayesian Likelihood (BLE) method by Hall and Wang (2005). The following points are worth noting:

- In terms of bias and RMSE, the estimator of the shape parameter proposed is outperforming those of the two 
concurrent methods.

- If the bias of the proposed estimator of the shape parameter is close to that of the other methods when the theoretical value of the shape parameter does not exceed 1.5, it should be noted that the MosLE method provides the smallest bias when the theoretical values of the shape parameter become large.

- Although the proposed estimator of the scale parameter is biased, it presents performances comparable to those of the other two methods in terms of RMSE.

\section{References}

Cheng, R. C. H., \& Iles, T. (1990). Embedded models in three-parameter distributions and their estimation. Journal of the Royal Statistical Society. Series B (Methodological), 52(1), 135-149. https://doi.org/10.1111/j.25176161.1990.tb01777.x

Cheng, R. C. H., \& Iles, T. C. (1987). Corrected maximum likelihood in non-regular problems. Journal of the Royal Statistical Society. Series B (Methodological), 49(1), 95-101. https://doi.org/10.1111/j.2517-6161.1987.tb01430.x

David, H. A., \& Nagaraja, H. (2003). Order statistics, Third Edition. Wiley Online Library. https://doi.org/10.1002/0471722162

Etienne Ouindllassida Jean Ouedraogo, B. S., \& Dossou-Gbete, S. (2017). On maximum likelihood estimation for the three parameter gamma distribution based on left censored samples. Science Journal of Applied Mathematics and Statistics, 5, 147.

Griffiths, A. (1980). Interval estimation for the three-parameter lognormal distribution via the likelihood function. Journal of the Royal Statistical Society. Series C (Applied Statistics), 29(1), 58-68.

Hall, P., \& Wang, J. (2005). Bayesian likelihood methods for estimating the end point of a distribution. Journal of the Royal Statistical Society, 67(5), 717-729. https://doi.org/10.1111/j.1467-9868.2005.00523.x

Hill, B. (1963). The three-parameter log-normal distribution and bayesian analysis of a point-source epidemic. J. Amer. Statist. Assoc., 58, 72-84. https://doi.org/10.1080/01621459.1963.10500833

Hunter, D., \& Lange, K. (2004). A tutorial on mm algorithms. The American Statistician, 58(1), 30-37. https://doi.org/10.1198/0003130042836

Lange, K.and Hunter, D., \& Yang, I. (2000). Optimization transfer using surrogate objective functions (with discussion). Journal of Computational and Graphical Statistics, 9(1), 1-20. https://doi.org/10.2307/1390605

Lange, K. (2013). Optimization,Second Edition, 95. Springer New York. https://doi.org/10.1007/978-1-4614-5838-8

Nagatsuka, H., \& Balakrishnan, N. (2012). An efficient method of parameter and quantile estimation for the threeparameter weibull distribution based on statistics invariant to unknown location parameter. Journal of Statistical Computation and Simulation, 142, 1-19.

Nagatsuka, H., Kamakura, T., \& Balakrishnan, N. (2013). A consistent method of estimation for the three-parameter weibull distribution. Computational Statistics and Data Analysis, 58, 210-226. https://doi.org/10.1016/j.csda.2012.09.005

R Core Team (2015). R: A language and environment for statistical computing. Retrieved from http://www.R-project.org/

Smith, R. (1985). Maximum likelihood estimation in a class of nonregular cases. Biometrika, 72(1), 67-90. https://doi.org/10.1093/biomet/72.1.67

Smith, R. L. (1994). Likelihood and modified likelihood estimation for distributions with unknown endpoints.

Vaida, F. (2005). Parameter convergence for em and mm algorithms. Statist. Sinica, 15, 831-840. 


\section{A. Appendix}

\section{A.1 Some basic inequalities}

Let $u, v, u^{\prime}$ and $v^{\prime}$ be strictly positive real numbers. The following assertions hold

1. $-\ln (v) \geq-\ln \left(v^{\prime}\right)-\frac{1}{v^{\prime}}\left(v-v^{\prime}\right)$

2. $-u v \geq-\frac{u^{2}}{2} \frac{v^{\prime}}{u^{\prime}}-\frac{v^{2}}{2} \frac{u^{\prime}}{v^{\prime}}$

3. $\ln (u+v) \geq \ln \left(u+v^{\prime}\right)+\frac{v^{\prime}}{u+v^{\prime}} \ln \left(\frac{v}{v^{\prime}}\right)$

As a consequence of the inequalities (1) and (2) stated above one has

$$
u \ln (v) \geq u \ln \left(v^{\prime}\right)+u-\frac{u^{2}}{2 u^{\prime}}-\frac{v^{\prime 2} u^{\prime}}{2 v^{2}}
$$

since $\ln (v)=-\ln \left(\frac{1}{v}\right) \geq \ln \left(v^{\prime}\right)-v^{\prime}\left(\frac{1}{v}-\frac{1}{v^{\prime}}\right)$.

\section{A.2 Proof of Proposition 3.1}

\section{Lemma A.1.}

$$
\begin{gathered}
-\ln \left(x_{r: n}-v\right) \geq-\ln \left(x_{r: n}-v^{\prime}\right)+\frac{v-v^{\prime}}{x_{r: n}-v^{\prime}} \\
-\lambda \ln (\beta) \geq-\lambda \ln \left(\beta^{\prime}\right)+\lambda-\frac{\lambda^{2}}{2 \lambda^{\prime}}-\lambda^{\prime} \frac{\beta^{2}}{2 \beta^{\prime 2}}
\end{gathered}
$$

Proof. By applying the convexity inequality to the $-\ln$ function one obtains

Moreover

$$
-\ln \left(x_{r: n}-v\right) \geq-\ln \left(x_{r: n}-v^{\prime}\right)+\frac{v-v^{\prime}}{x_{r: n}-v^{\prime}}
$$

$$
\begin{aligned}
-\lambda \ln (\beta) & \geq \lambda\left\{-\ln \left(\beta^{\prime}\right)-\frac{1}{\beta^{\prime}}\left(\beta-\beta^{\prime}\right)\right\} \\
& \geq-\lambda \ln \left(\beta^{\prime}\right)+\lambda-\frac{1}{\beta^{\prime}}\left\{\frac{\lambda^{2}}{2} \frac{\beta^{\prime}}{\lambda^{\prime}}+\frac{\beta^{2}}{2} \frac{\lambda^{\prime}}{\beta^{\prime}}\right\} \\
& \geq-\lambda \ln \left(\beta^{\prime}\right)+\lambda-\frac{\lambda^{2}}{2 \lambda^{\prime}}-\lambda^{\prime} \frac{\beta^{2}}{2 \beta^{\prime 2}}
\end{aligned}
$$

\section{Lemma A.2.}

$$
\lambda \log \left(x_{r: n}-v\right) \geq \lambda \ln \left(x_{r: n}-v^{\prime}\right)+\left[\frac{x_{1: n}-v^{\prime}}{x_{r: n}-v^{\prime}}\right] \lambda-\left[\frac{x_{1: n}-v^{\prime}}{x_{r: n}-v^{\prime}}\right] \frac{\lambda^{2}}{2 \lambda^{\prime}}-\left[\frac{x_{1: n}-v^{\prime}}{x_{r: n}-v^{\prime}}\right] \frac{\left(x_{1: n}-v^{\prime}\right)^{2}}{\left(x_{1: n}-v\right)^{2}} \frac{\lambda^{\prime}}{2}
$$

Proof. use inequality A.1

Appealing the Lemma above one has:

$$
\begin{aligned}
Q_{1}\left(\theta \mid \theta^{\prime},\left(x_{i: n}\right)_{i=1: n}\right)= & (n-1) \ln \lambda+(n-1)\left[-\lambda \ln \left(\beta^{\prime}\right)+\lambda-\frac{\lambda^{2}}{2 \lambda^{\prime}}-\lambda^{\prime} \frac{\beta^{2}}{2 \beta^{\prime 2}}\right] \\
& -\sum_{r=2}^{n} \ln \left(x_{r: n}-v^{\prime}\right)+\sum_{r=2}^{n} \frac{v-v^{\prime}}{x_{r: n}-v^{\prime}} \\
& +\lambda \sum_{r=2}^{n} \ln \left(x_{r: n}-v^{\prime}\right)+\sum_{r=2}^{n}\left[\frac{x_{1: n}-v^{\prime}}{x_{r: n}-v^{\prime}}\right] \lambda-\sum_{r=2}^{n}\left[\frac{x_{1: n}-v^{\prime}}{x_{r: n}-v^{\prime}}\right] \frac{\lambda^{2}}{2 \lambda^{\prime}} \\
& -\sum_{r=2}^{n}\left[\frac{x_{1: n}-v^{\prime}}{x_{r: n}-v^{\prime}}\right] \frac{\left(x_{1: n}-v^{\prime}\right)^{2}}{\left(x_{1: n}-v\right)^{2}} \frac{\lambda^{\prime}}{2}
\end{aligned}
$$


After rearangements, the result hold.

\section{A.3 Proof of Proposition 3.2}

\section{Lemma A.3.}

$$
\begin{aligned}
\lambda \ln \left(x_{r: n}-v\right)-\lambda \ln \beta & \leq \lambda \ln \left(x_{r: n}-v^{\prime}\right)-2 \lambda+\frac{\lambda^{2}}{\lambda^{\prime}}-\lambda \log \beta^{\prime}+\frac{1}{\beta^{2}} \frac{\beta^{\prime 2} \lambda^{\prime}}{2} \\
& +\left(x_{r: n}-v\right)^{2} \frac{\lambda^{\prime}}{2\left(x_{r: n}-v^{\prime}\right)^{2}}
\end{aligned}
$$

Proof. since $-\ln (x)+\ln \left(x^{\prime}\right) \geq 1-\frac{x}{x^{\prime}}$, one can derive

$$
\lambda \ln \left(x_{r: n}-v\right) \leq \lambda \ln \left(x_{r: n}-v^{\prime}\right)-\lambda+\frac{\lambda\left(x_{r: n}-v\right)}{\left(x_{r: n}-v^{\prime}\right)}
$$

From

$$
\frac{\lambda\left(x_{r: n}-v\right)}{\left(x_{r: n}-v^{\prime}\right)} \leq \frac{\lambda^{2}}{2 \lambda^{\prime}}+\left(x_{r: n}-v\right)^{2} \frac{\lambda^{\prime}}{2\left(x_{r: n}-v^{\prime}\right)^{2}}
$$

one has

$$
\lambda \ln \left(x_{r: n}-v\right) \leq \lambda \ln \left(x_{r: n}-v^{\prime}\right)-\lambda+\frac{\lambda^{2}}{2 \lambda^{\prime}}+\left(x_{r: n}-v\right)^{2} \frac{\lambda^{\prime}}{2\left(x_{r: n}-v^{\prime}\right)^{2}}
$$

Moreover

$$
\begin{aligned}
-\lambda \ln \beta & =\lambda \ln \frac{1}{\beta} \\
& \leq-\lambda \ln \beta^{\prime}-\lambda+\frac{\lambda^{2}}{2 \lambda^{\prime}}+\frac{1}{\beta^{2}} \frac{\beta^{\prime 2} \lambda^{\prime}}{2}
\end{aligned}
$$

After rearangements, the result hold.

Let's denote

$$
\begin{gathered}
A(\lambda)=\lambda \ln \left(x_{r: n}-v^{\prime}\right)-2 \lambda+\frac{\lambda^{2}}{\lambda^{\prime}}-\lambda \ln \beta^{\prime} \\
B(\beta)=\frac{1}{\beta^{2}} \frac{\beta^{2} \lambda^{\prime}}{2}
\end{gathered}
$$

and

$$
C(v)=\left(x_{r: n}-v\right)^{2} \frac{\lambda^{\prime}}{2\left(x_{r: n}-v^{\prime}\right)^{2}}
$$

Using the fact that $-\left(\frac{x_{r: n}-v}{\beta}\right)^{\lambda}=-\exp \left(\lambda \ln \left(x_{r: n}-v\right)-\lambda \ln \beta\right)$ and by appealing lemma A.3 one has

$$
-\left(\frac{x_{r: n}-v}{\beta}\right)^{\lambda} \geq-\exp (A(\lambda)) \exp (B(\beta)) \exp (C(v))
$$

By the arithmetic-geometric mean inequality, one derived that 


$$
\begin{aligned}
-\left(\frac{x_{r: n}-v}{\beta}\right)^{\lambda} & \geq-\frac{\exp (3 A(\lambda))}{3} \frac{\exp \left(B\left(\beta^{\prime}\right)\right) \exp \left(C\left(v^{\prime}\right)\right)}{\exp \left(2 A\left(\lambda^{\prime}\right)\right)} \\
& -\frac{\exp (3 B(\beta))}{3} \frac{\exp \left(A\left(\lambda^{\prime}\right)\right) \exp \left(C\left(v^{\prime}\right)\right)}{\exp \left(2 B\left(\beta^{\prime}\right)\right)} \\
& -\frac{\exp (3 C(v))}{3} \frac{\exp \left(A\left(\lambda^{\prime}\right)\right) \exp \left(B\left(\beta^{\prime}\right)\right)}{\exp \left(2 C\left(v^{\prime}\right)\right)}
\end{aligned}
$$

By replacing $A(\lambda), B(\beta)$ and $C(\nu)$ with their value and after rearrangement one has

$$
l_{2}\left(\theta \mid x_{2: n}, \cdots, x_{n: n}\right)=-\sum_{r=2}^{n}\left(\frac{x_{r: n}-v}{\beta}\right)^{\lambda}-\left(\frac{x_{2: n}-v}{\beta}\right)^{\lambda} \geq Q_{2}\left(\theta \mid \theta^{\prime},\left(x_{i: n}\right)_{i=1: n}\right)
$$

where $Q_{2}\left(\theta \mid \theta^{\prime},\left(x_{i: n}\right)_{i=1: n}\right)$ is given in Proposition 3.1

\section{A.4 Proof of Proposition 3.3}

Lemma A.4. Let denote $\Pi\left(\theta \mid x_{2: n}\right)=\left(\frac{x_{2: n}-\gamma}{\beta}\right)^{\lambda}$. One has

$$
\begin{aligned}
-\Pi\left(\theta \mid x_{2: n}, \theta^{\prime}\right) \geq & -\frac{\exp \left[3 \lambda \ln \left(x_{2: n}-v^{\prime}\right)-6 \lambda+\frac{3 \lambda^{2}}{\lambda^{\prime}}-3 \lambda \ln \beta^{\prime}\right]}{3 \exp \left(2 \lambda^{\prime} \ln \left(x_{2: n}-v^{\prime}\right)-3 \lambda^{\prime}-2 \lambda^{\prime} \ln \beta^{\prime}\right)} \\
& -\frac{\exp \left(\frac{3}{\beta^{2}} \frac{\beta^{\prime 2} \lambda^{\prime}}{2}\right)}{3} \exp \left(\lambda^{\prime} \ln \left(x_{2: n}-v^{\prime}\right)-\frac{3}{2} \lambda^{\prime}-\lambda^{\prime} \ln \beta^{\prime}\right) \\
& -\frac{\exp \left(\left(x_{2: n}-v\right)^{2} \frac{3 \lambda^{\prime}}{2\left(x_{2: n}-v^{\prime}\right)^{2}}\right)}{3} \exp \left(\lambda^{\prime} \ln \left(x_{2: n}-v^{\prime}\right)-\frac{3}{2} \lambda^{\prime}-\lambda^{\prime} \ln \beta^{\prime}\right)
\end{aligned}
$$

Proof. Direct application of inequality (A.2)

\section{Lemma A.5.}

$$
l_{3}\left(\theta \mid x_{2: n}\right)=\ln \left(\exp \left(\Pi\left(\theta \mid x_{2: n}\right)\right)-1\right) \geq a \ln \left(\Pi\left(\theta \mid x_{2: n}\right)\right)+b \Pi\left(\theta \mid x_{2: n}\right)+c
$$

where

$$
\begin{gathered}
a=\frac{\Pi^{2}\left(\theta^{\prime} \mid x_{2: n}\right) \exp \left(\Pi\left(\theta^{\prime} \mid x_{2: n}\right)\right)}{\left[\exp \left(\Pi\left(\theta^{\prime} \mid x_{2: n}\right)\right)-1\right]^{2}}+\frac{\Pi\left(\theta^{\prime} \mid x_{2: n}\right) \exp \left(\Pi\left(\theta^{\prime} \mid x_{2: n}\right)\right)}{\exp \left(\Pi\left(\theta^{\prime} \mid x_{2: n}\right)\right)-1} \\
b=-\frac{\Pi\left(\theta^{\prime} \mid x_{2: n}\right) \exp \left(\Pi\left(\theta^{\prime} \mid x_{2: n}\right)\right)}{\exp \left(\Pi\left(\theta^{\prime} \mid x_{2: n}\right)\right)-1}
\end{gathered}
$$

and

$$
c=\ln \left(\exp \left(\Pi\left(\theta^{\prime} \mid x_{2: n}\right)\right)-1\right)-a \ln \left(\Pi\left(\theta^{\prime} \mid x_{2: n}\right)\right)-b \Pi\left(\theta^{\prime} \mid x_{2: n}\right)
$$

Proof. Results from the convexity of the function $x \rightarrow \ln (\exp (x)-1)$.

\section{Lemma A.6.}

$$
\begin{aligned}
a \ln \left(\Pi\left(\theta \mid x_{2}\right)\right) & \geq a \lambda\left\{\ln \left(x_{2: n}-v^{\prime}\right)-\ln \left(\beta^{\prime}\right)+1+\left[\frac{x_{1: n}-v^{\prime}}{x_{2: n}-v^{\prime}}\right]\right\}-a\left[1+\frac{x_{1: n}-v^{\prime}}{x_{2: n}-v^{\prime}}\right] \frac{\lambda^{2}}{2 \lambda^{\prime}} \\
& -a\left[\frac{x_{1: n}-v^{\prime}}{x_{2: n}-v^{\prime}}\right] \frac{\lambda^{\prime}\left(x_{1: n}-v^{\prime}\right)^{2}}{2\left(x_{1: n}-v\right)^{2}}-a \lambda^{\prime} \frac{\beta^{2}}{2 \beta^{\prime 2}}
\end{aligned}
$$


Proof.

$$
a \ln \left(\Pi\left(\theta \mid x_{2}\right)\right)=a\left[\lambda \ln \left(x_{2: n}-v\right)-\lambda \ln \beta\right]
$$

Applying lemma A.1 andA.2, the result hold, since $a>0$

\section{Lemma A.7.}

$$
\begin{aligned}
b \Pi\left(\theta \mid x_{2}\right) \geq & \frac{b \exp \left[3 \lambda \ln \left(x_{2: n}-v^{\prime}\right)-6 \lambda+\frac{3 \lambda^{2}}{\lambda^{\prime}}-3 \lambda \ln \beta^{\prime}\right]}{3 \exp \left(2 \lambda^{\prime} \ln \left(x_{2: n}-\nu^{\prime}\right)-3 \lambda^{\prime}-2 \lambda^{\prime} \ln \beta^{\prime}\right)} \\
& +\frac{b \exp \left(\frac{3}{\beta^{2}} \frac{\beta^{2} \lambda^{\prime}}{2}\right)}{3} \exp \left(\lambda^{\prime} \ln \left(x_{2: n}-v^{\prime}\right)-\frac{3}{2} \lambda^{\prime}-\lambda^{\prime} \ln \beta^{\prime}\right) \\
& +\frac{b \exp \left(\left(x_{2: n}-v\right)^{2} \frac{3 \lambda^{\prime}}{2\left(x_{2: n}-v^{\prime}\right)^{2}}\right)}{3} \exp \left(\lambda^{\prime} \ln \left(x_{2: n}-v^{\prime}\right)-\frac{3}{2} \lambda^{\prime}-\lambda^{\prime} \ln \beta^{\prime}\right)
\end{aligned}
$$

Proof. Applying lemma A.4 and taken acount that $b<0$, the result hold.

To complete the proof of the Proposition 3.3, remember that $l_{3}\left(\theta \mid x_{2: n}\right)=\ln \left(\exp \left\{\left(\frac{x_{2: n}-v}{\beta}\right)^{\lambda}\right\}-1\right)$. Appealing precedents Lemmas, the result hold.

\section{Copyrights}

Copyright for this article is retained by the author(s), with first publication rights granted to the journal.

This is an open-access article distributed under the terms and conditions of the Creative Commons Attribution license (http://creativecommons.org/licenses/by/4.0/). 